\title{
Prevalence of depressive symptoms and associated factors among people with Tuberculosis in Ethiopia: meta-analysis
}

Berhanu Boru Bifftu ( $\sim$ berhanuboru@gmail.com )

University of Gondar https://orcid.org/0000-0002-4229-847X

Bewket Tadesse Tiruneh

University of Gondar

Yonas Deressa Guracho

Bahir Dar University

Berihun Assefa Dachew

University of Gondar

Research article

Keywords: Depressive symptoms, Ethiopia, Factors, Tuberculosis

Posted Date: November 11th, 2019

DOI: https://doi.org/10.21203/rs.2.17054/v1

License: (a) (i) This work is licensed under a Creative Commons Attribution 4.0 International License.

Read Full License 


\section{Abstract}

Tuberculosis (TB) is a chronic infectious disease caused by mycobacterium tuberculosis. Co-morbid depression among individual with TB is common public health concern that adversely affect the psychological well being of the patient. Thus, the aims of this study were to determine the magnitude of depression and its associated factors among people with TB in Ethiopia. Methods: Databases including: PubMed/Medline, SCOPUS, and HINARI were searched. Weighted inverse variance random-effects model was used to calculate the pooled prevalence of depression. The heterogeneity between studies was measured by the index of heterogeneity (12 statistics) test. Funnel plots and Egger's test were used to determine publication bias. Sensitivity test and subgroup analysis were also performed to identify influential study and to account for heterogeneity. Results: In this study, a total of 6430individuals with TB were included from 15 studies. The pooled prevalence of depression was $46 \%$ (95\% Cl; $40 \%-53 \%$ ). Tests of heterogeneity was significant $(I 2=96.33 \%$ and $p<0.001)$. However, funnel plots and Egger's test showed no evidence of publication bias. Being female, low income, substance use, perceived stigma, extra-pulmonary TB and treatment phase were factors associated with depressive symptoms.

Conclusion: In Ethiopia, around half of the TB patients had depressive symptoms. Thus, authors' suggest the need of mental health screening for people with TB particularly for substance users and socioeconomic disadvantage TB patients. Authors' also suggest the needs of improving awareness of TB.

\section{Background}

Tuberculosis (TB) is a chronic infectious disease caused by mycobacterium tuberculosis(1). TB affects one-third of the world's population, of which majority are from developing countries like Ethiopia (2, 3).According to WHO 2018 data, Ethiopia was among the top 14 countries with burden of TB, TB-HIV coinfection and multi-drug resistant TB(3).

Mental illness such as depression, anxiety, and somatic symptom are growing global public concerns with an estimated lifetime prevalence of one in three individuals (4).Co-morbid mental illness among individual with TB is also another common public concern (5-7).Previous studies revealed that more than $50 \%$ of people with TB had one or more of mental illness such as depression, anxiety, somatic symptoms or their combinations $(5,7)$. Because depression mimics and magnifies the symptoms of TB such as poor appetite, loss of weight and easy fatigability(8). Studies showed that depression is the silent driver of global TB epidemic and responsible for the emergence of Multi Drug Resistant TB $(6,8)$.. Co-morbid depression among people with TB is an obstacle for the achievement of Tb eradication by its association with delays in tuberculosis diagnosis and treatment, poor treatment outcomes, disability, poor quality of life, treatment failure, and death (7). This co-morbidity depression is related to the nature of the illness, biological factors, social (stigma, poverty), and behavioral factors (substance use)(5-9). In Ethiopia, epidemiological evidence showed that the prevalence of depression among individual with TB ranged from $18 \%$ to $67 \%$ (9-21). This co-morbid depression was associated with poor treatment adherence, MRD-TB, suicide, substance use and poor quality of life $(9,10,22)$. Although, these individual 
studies are important, the variation in reported prevalence of depression and its determinant factors need comprehensive up to date information. However, to the authors' knowledge, there is no systematic review and meta-analysis that address this need. Therefore, the present systematic review and meta-analysis aims (i) to determine the pooled magnitude of depression and (ii) to synthesize the factors associated with depression among people with TB in Ethiopia.

\section{Methods}

\section{Search strategy}

We systematically searched databases such as: PubMed/Medline, SCOPUS, HINARI, AIM and AJOL were searched. A comprehensive search strategy was formulated by the combination of Medical Subject Headings (MeSH) thesaurus, text words, and appropriate Boolean Operators ("AND" and "OR"). No time and language restrictions were imposed on the search strategy. Reference lists of all articles and Google Scholar were also performed. All searches were performed until October 12, 2019. The full electronic search strategy for PubMed/MEDLINE database was ((tubercul* [MeSH Terms]) OR (tubercul*) OR ('lung tuberculosis' [MeSH Terms]) OR ('lung tuberculosis') OR ('pulmonary consumption' [MeSH Terms]) OR ('pulmonary consumption') OR ('consumption, pulmonary' [MeSH Terms]) OR ('consumption, pulmonary') OR ('Tuberculosis' [MeSH Terms]) OR ('Tuberculosis') OR ('TB'[MeSH Terms]) OR ('TB')) And ((depressive disorders [MeSH Terms]) OR (depressive disorders) OR (depressive symptoms [MeSH Terms]) OR (depressive symptoms) AND ((associated factors [MeSHTerms]) OR (associated factors) OR (determinant factors [MeSH Terms]) OR (determinant factors) AND ((epidemiology [MeSH Terms]) OR (epidemiology) OR (prevalence [MeSH Terms]) OR (prevalence) OR (incidence [MeSH Terms]) OR (incidence) OR (magnitude [MeSH Terms]) OR (magnitude)) AND Ethiopia. The report of this systematic review and meta-analysis was conducted according to the Preferred Reporting Items for Systematic Review and Meta-Analysis (PRISMA) (23).

\section{Selection of studies}

All studies retrieved through search strategy were imported in to EndNote X7 (Thomson Reuters, New York, USA) and duplications were removed. The title and abstracts of the remaining articles were assessed independently by two reviewers (BBB and YDG) and disagreements were resolved discussion.

\section{Eligibility Criteria}

Setting: This review included studies carried out in Ethiopia.

Participants: This review included data from people with TB.

Outcome measure: This systematic review and meta-analysis have two main outcomes. These are (i) the pooled prevalence of depression and (ii) factors associated with depression. The prevalence of depression was calculated by dividing the number of people with co-morbid TB-depression to the total 
number of people with TB included in the study (total sample size) multiplied by 100 . For the associated factors, the reported odds ratio, $95 \%$ confidence interval and $p$-value were used.

Study design: Observational studies (cross-sectional and cohort/longitudinal) were included. Studies that focused on case reports, conference and abstracts were excluded.

\section{Data extraction}

Data were extracted from the eligible studies using a pre-conceived data abstraction form by two independent reviewers (BBB and YDG). The extracted data include: name of the first author's last name, year of publication, study area/region, study design, assessment tool, sample size, number of cases/prevalence and associated factors.

\section{Quality assessment}

The Newcastle-Ottawa quality assessment tool, adapted for cross-sectional studies (24) was used for quality assessment. This tool had three main parts (selection, comparability and outcome). The first part (selection) has five stars and assesses the methodological quality of the study. The second part of the tool evaluates the comparability of the study. The third part of the tool assesses the quality of the original article's outcome with respect to the statistical analysis. Individual paper was graded with score ranged from zero to ten stars. The overall quality of each study was determined using the sum score of each star of the three parts. If the overall score of a paper was $\geq 6$ out of 10 , it is categorized as good quality and if the score fulfilling $50 \%$ of quality assessment criteria, it is medium and for score $\leq 4$, it is defined as poor quality.

\section{Data synthesis and statistical analysis}

The extracted data were entered into a Microsoft Excel Database and then imported into STATA version 14 (Stata Corp LLC, Texas, USA) software with packages of Meta-analysis for analysis. Meta-analysis was performed using STATA 14. "Metaprop" command with random-effects models was used to calculate the pooled effect size at 95\% confidence intervals (25) with the Dersimonian and Laird method based transformed values and their variance (26). The Freeman-Tuckey variant of the arcsine square root transformation of proportions was fitted to avoid variance instability when handling proportions close to one (27). Heterogeneity between studies was measured by the index of heterogeneity $\left(1^{2}\right.$ statistics) test (28). $I^{2}$ statistics values of $25 \%, 50 \%$ and $75 \%$ was used as low, medium and high heterogeneity respectively. Publication bias was examined by the visual inspection of funnel plot (29) and egger test (30) ( $p$-value $<0.05)$. Sensitivity analyses was conducted to examine influential studies(31). Sub-group analysis was performed by sample size, study design, publication year, quality of studies and study setting. For the analysis of associated factors, the reported odds ratio with $95 \%$ confidence interval and p-value were used. Meta-analysis of the associated factors was performed for those factors with at least two studies reported the same associated factors. 


\section{Results}

The initial database literature search resulted in 619 published articles. Additional, 23 studies were found from other source and resulted in 642overall articles. Of these articles, 424 were excluded during the initial assessments based on their title and abstract reading and found to be irrelevant. Of the remaining 218 studies, 119 articles were excluded because of different outcome and unclear results. Finally, 84 papers were excluded as results of eligibility criteria. Then, the remained 15 studies were included in the systematic review and meta-analysis (Fig. 1).

\section{Study characteristics}

In this study, a total of 6430 study participants were included from four different regions: Addis Ababa $(n=5)$, Oromia $(n=5)$ and SNNP $(n=5)$. Majority $(73 \%)$ of included studies were cross-sectional and published between 2010 and September 2019. The sample sizes of included studies ranged from 97 to 817 , with the response rate of $78-100 \%$. All studies utilized a validated depression assessment tool. Of this, majority (46\%) was used PHQ-9 ( $n=7)$ and $40 \%$ K-10 ( $n=6)$ (Table: 1$)$.

\section{Quality of included studies}

The overall quality score of included studies ranged from 4 to 9 . Of these, eleven studies had good quality and the remaining four studies had fair quality (Supplementary Table: 1).

\section{Test of heterogeneity, publication bias and subgroup analysis}

The tests of heterogeneity demonstrated significant heterogeneity $\left(I^{2}=96.33 \%\right.$ and $\left.p<0.001\right)$. But, there was no evidence of publication bias from the visual inspection of the funnel plot (Fig. 2) and Egger's test $(P=0.06)]$. The results of sensitivity analysis also showed that none of the point estimates was outside of the overall $95 \%$ confidence interval confirming that there was no influential study. Thus, in this study, the pooled prevalence of depression based on the 15 studies could be important.

Subgroup analysis demonstrated higher pooled prevalence of depression among studies with longitudinal study design (pooled prevalence $=47 \%$ (95\% Cl: 32\%-62\%), larger sample size (pooled prevalence $=49 \%$ (95\% Cl: 44\%-55\%), good quality studies[pooled prevalence $=52 \%(95 \% \mathrm{Cl}: 46 \%-58 \%)$, publication year 2015-2019 [pooled prevalence $=49 \%(95 \% \mathrm{Cl}: 43 \%-56 \%)$ ] and among studies conducted in Oromia. From the study level characteristics of subgroup analysis by study design, sample size, study quality, publication year and study setting were significant, which implies the presence of other characteristics that may contribute for the heterogeneity (Table 2).

\section{Prevalence of depression}

The overall pooled prevalence of depression was found to be $46 \%$ (95\% Cl; $40 \%-53 \%)$ (Fig: 2). 


\section{Factors associated with depression}

Based on this study, factors such as: socio-demographic factors (sex and income), social factors (perceived stigma), behavioral factors (substance use) and clinical factors (extra-pulmonary TB and TB treatment phase) were associated with depression (Table: 3 ).

Socio-demographic factors and depression: In this study, the pooled effect of four studies $(12,16,19,32)$ showed that females were around two times (AOR=1.6;95\%, 1.10-2.23) more likely experienced depression as compared to males. Moreover, those TB-patients with low socio-economic status or income $(9,11,14)$ were three times (AOR=3.0;95\%, 1.16-4.92) more likely to have depression symptoms compared with those with high income category.

Social factors and depression: In this study, those participant with history of perceived stigma were more than two times (AOR=2.2;95\%, 1.67-6.40) $(11,12,14,15,32)$ more likely experienced depression compared with those who did not experience perceived stigma.

Behavioral factors and depression: In this study, use of substance such as: alcohol and cigarette $(9,11$, 12) were the risk factors for depression with the pooled estimate of 3.2 (AOR=3.2; 95\%, 1.80-4.45).

Clinical factors and depression: Clinical factors such as extra pulmonary TB $(11,32)$ (AOR=2.1; $95 \% 1.48$ $2.78)$ and intensive phase of T-treatment $(A O R=1.6 ; 95 \%, 1.10-2.09)(11,12,14)$ were also associated with depression.

\section{Discussion}

This systematic review and meta-analysis estimated the epidemiological evidence of depression and its determinant factors among people with Tuberculosis. In this study, the prevalence of depression among people with Tuberculosis was found to be $46 \%$ (95\% Cl; $40 \%-53 \%)$. Even though similar study is scarce, this result is higher than the reported prevalence of depression in the general community of Ethiopia (32). For example, a previous systematic review and meta-analysis using data from 13 studies reported $20.5 \%$ (95\% Cl; $16.5 \%-24.4 \%$ ) of depression in the general community (33). This showed that people with tuberculosis is more than two times more likely to suffer with depression as compared to the general population. In line with the present study, a comparative community based study using data from 48 lowand middle-income countries revealed that higher prevalence of depressive episode among those people with tuberculosis as compared to the general population (5).The increased prevalence of depression among people with TB may be due to the negative psycho-social effect of TB and its associated drug side effect (citation). In consistent with our study, another similar systematic review and meta-analysis among people with TB using data from 31 studies in 11 different countries reported a prevalence of $48.9 \%$ (95\% Cl 48.3\%-49.6\%) (8). Another meta-analysis also reported a pooled prevalence of $50 \%$ (19\%$81 \%)$ using data of base line report (34). 
In Ethiopia, there is a strong political commitment to control TB and the burden of mental illness, and result in decreasing trend in TB mortality and improved experiences in implementation of mental health integration; however the priority focus is given to curative services rather than mental, neurological and substance use disorders.

In this study, we found that being female was around two times (AOR=1.6; 95\%, 1.10-2.23) more likely experienced depression as compared to male. This is consistent with previous studies. Similarly, those individual with low socio-economic status were three times (AOR=3; 95\%, 1.16-4.92) more likely reported depression. This may be associated with individual's inability to fulfill their needs like food, drug and other, which is the cause for stress that in turn to depression. Studies showed that people living in low socio-economic status have a greater risk of exposure to precipitating factors of depression such as violence, stigma, discrimination and lacking adequate health care information or education $(6,8)$.

In this study, those individual with history of perceived stigma were more likely experience depression. This may be due to the fear associated with the knowledge of TB transmission, that in turn for selfstigma which is an important risk for depression. In this study, use of substance was associated with depression. This may be due to people's use of substance as a coping mechanism of depression and TB burden like poverty. Type of TB (extra pulmonary) and intensive phase of TB-treatment were also associated with depression. This may be related to individual's perceived severity of the illness and anti Tb drug side effect. Evidence showed that

The associations of several treatment drug with psychiatric illness such as: cycloserine (CS), isoniazid $(\mathrm{INH})$, ethionamide, ciprofloxacin, ethambutol, rifampicin (RMP), and the fluoroquino-lones $(5,6,35)$. That is why the WHO End TB Strategy 2015-35 calls for the integration TB- mental health treatment for the management of co-morbid psychological distress (36). Thus, the results of the preset study imply high magnitude of depression among people with TB. Moreover, this study also implies the adverse effect of TB on an individuals' socio-economic status, behavior like substance use and individuals' perception of the illness for the psychological well being TB patients. This may suggest the needs of evaluating the effectiveness of health care system in addressing the co-morbid mental illness.

\section{Strengths and limitations of the study}

To our knowledge, this is the first systematic review and meta-analysis about the prevalence of alcohol use and its associated factors among Ethiopia pregnant women. To manage heterogeneity and bias; First, we used appropriate model (a random effect model) to control the effects of the observed heterogeneity. Secondly, we conducted a leave one out sensitivity analysis to identify influential study. Third, we conducted sub-group analysis to account for heterogeneity. Forth, funnel plot and egger test was performed to identify small study effect. However, limitations such as: use of reference lists and Google Scholar may have the possibility of having some overlooked articles.

\section{Conclusion}


In Ethiopia, around half of people with TB had an experience of depressive symptom. Being female, low income, substance use, perceived stigma, extra-pulmonary TB and treatment phase were factors associated with depressive symptom. Thus, authors' suggest the need of screening people with TB particularly for those with low income, negative perceptions of TB and history of substance use during the initial phase of the treatment. Authors' also suggest the needs of improving awareness of TB.

\section{Abbreviations}

Cl: Confidence Interval, DSM-IV: Diagnostic and Statistical Manual of Mental Disorders $4^{\text {th }}$ edition, K-10: Kessler Psychological/Mental Distress Scale, EMBASE: ExcerptaMedica database, 12: Index of heterogeneity, ICD-10: Tenth Revision of the International Statistical Classification of Diseases and Related Health Problems, HADS: Hospital Anxiety and Depression Scale, PHQ-9: Patient Health Questionnaire-9, MEDLINE: Medical Literature Analysis and Retrieval System Online, MeSH: Medical Subject Headings, OR: Odd Ratio, PRISMA: Preferred Reporting Items for Systematic Review and MetaAnalysis, PROSPERO: International Prospective Register of Systematic Reviews, WHO: World Health Organization.

\section{Declarations}

\section{Ethics approval and consent to participants}

Not applicable.

\section{Consent for publication}

Not applicable.

\section{Availability of data}

Not applicable.

\section{Competing interests}

None declared.

\section{Funding}

This research received no grant from any funding agency in the public, commercial or not-for-profit sectors.

\section{Authors' contributions}

BBB designed the systematic review and meta-analysis in collaboration with BAD, BTT and YDG. BBB developed the search strategy and drafted the protocol. BAD, BTT and YDG improved the drafted 
systematic review and meta-analysis. BBB, YDG and BAD provided their expertise to the section of mental illness (depression) and methodological section. BBB and YDG performed search strategy and conducted data selection and extraction. All authors were involved in data analysis and interpretation of the results and write up the manuscript. All authors have read and approved the final manuscript.

\section{Acknowledgements}

Authors' gratitude goes to University of Gondar for office arrangement and motivational support to conduct this systematic review and meta-analysis.

\section{Authors' information's}

${ }^{1}$ University of Gondar College of Medicine and Health Science, School of Nursing, Gondar, Ethiopia

${ }^{2}$ Bahar Dar University, College of Medicine and Health Science, Department of psychiatry Ethiopia.

${ }^{3}$ Department of Epidemiology and Biostatistics, Institute of Public Health, College of Medicine and Health Sciences, University of Gondar, Ethiopia.

${ }^{4}$ The University of Queensland, Institute for Social Science Research, Brisbane, QLD 4068, Australia

\section{References}

1. K Z. Tuberculosis: a global health problem. J Health Popul Nutr. 2010;28(2):111.

2. health FDRoEMo. GUIDELINES FOR MANAGEMENT OF TB, DR-TB AND LEPROSY IN ETHIOPIA SIXTH EDITION November 2017Addis Ababa.

3. Organization WH. Global tuberculosis report: profiles of high burden countries, annual report. Geneva; 2018.

4. Steel Z MC, Iranpour $\mathrm{C}$, et al The global prevalence of common mental disorders: a systematic review and meta-analysis 1980-2013. Int J Epidemiol. 2014;43(2):476-93.

5. al Ke. Depression comorbid with tuberculosis and its impact on health status: cross-sectional analysis of community-based data from 48 low- and middle-income countries BMC Medicine 2017;15(209).

6. al ACSe. Addressing the tuberculosis-depression syndemic to end the tuberculosis epidemic. Int $J$ Tuberc Lung Dis. 2017;21(8):852-61.

7. Dachew BA, Scott JG, Betts K, Mamun A, Alati R. Hypertensive disorders of pregnancy and the risk of offspring depression in childhood: Findings from the Avon Longitudinal Study of Parents and Children. Dev Psychopathol. 2019:1-7.

8. al ASe. Depression: a silent driver of the global tuberculosis Epidemic. World Psychiatry 2014;13(3).

9. Habteyes HH SD, Garmaroudi G, Tol A, Yekaninejad MS, Ejeta LT et al : . Psychological distress and its effect on tuberculosis treatment outcomes in Ethiopia. Global Health Action 2015;8(1):29019. 
10. Ambaw F MR, Hanlon C, Medhin G, Alem A. Impact of untreated depression on tuberculosis treatment outcomes, disability, and quality of life in Ethiopia: a cohort study. WHO Bulletin. 2018;96:245-55.

11. Dachew BA, Scott JG, Mamun A, Alati R. Pre-eclampsia and the risk of attention-deficit/hyperactivity disorder in offspring: Findings from the ALSPAC birth cohort study. Psychiatry Res. 2019;272:392-7.

12. Duko B GA, Ayano G: . Prevalence and correlates of depression and anxiety among patients with tuberculosis at Wolaita Sodo University Hospital and Sodo Health Center, WolaitaSodo, South Ethiopia, Cross sectional study. BMC Psychiatry 2015;15:214.

13. Adem A TM, Mohammed M: . The Prevalence and Pattern of Depression in Patients with Tuberculosis on Follow up at Jimma University Specialized Hospital and Jimma Health Center. Medicine Science | International Medical. Journal 2014, 3(1):955. 2014;3(1):955.

14. Amare D MT, Yohannes Hailmichael, Ludwig Apers, Gemeda Abebe, Luc Duchateau et al : . Common mental disorders in TB/HIV coinfected patients in Ethiopia BMC Infectious Diseases 2010;10(201):17.

15. al Me. Depression and associated factors among patients with tuberculosis in Ethiopia: a crosssectional study. Neuropsychiatric Disease and Treatment 2019;15:1887-93.

16. Ambaw F MR, Hanlon C, Alem A. Burden and presentation of depression among newly diagnosed individuals with TB in primary care settings in Ethiopia. BMC Psychiatry. 2017;17(1):57.

17. Molla A MA, Derjaew H, Kerebih H. . Suicidal ideation, attempt, and associated factors among patients with tuberculosis in Ethiopia: a cross-sectional study. Psychiatry Journal Volume 2019, Article ID 4149806, 10 pages https://doiorg/101155/2019/4149806. 2019.

18. al Ae. Do common mental disorders decline over time in TB/HIV co-infected and HIV patients without TB who are on antiretroviral treatment? BMC Psychiatry 2013;13(174):1-6.

19. al FAe. Is depression associated with pathways to care and diagnosis delay in people with tuberculosis in Ethiopia? Global Mental Health. 2019;6(20):1-11.

20. al YMe. Illness Perception, Coping Strategies and Psychosocial Adjustments among Adult TB Patients in Northwestern Ethiopia. Addis Ababa University. 2018:142.

21. Tola HH SD, Tol A, Garmaroudi G, Yekaninejad MS, Kebede A, et al. Psychological and Educational Intervention to Improve Tuberculosis Treatment Adherence in Ethiopia Based on Health Belief Model: A Cluster Randomized Control Trial. PLoS ONE 2016;11(5):1-15.

22. Habteyes HH GG, Shojaeizadeh D, Tol A, Yekaninejad MS, Ejeta LT et al : . The effect of psychosocial factors and patients' perception of tuberculosis treatment non-adherence in Addis Ababa, Ethiopia. . Ethiopian Journal of Health Sciences 2017;27(5):447.

23. Shamseer $L M D$, Clarke $M$, et al. Preferred reporting items for systematic review and meta-analysis protocols (PRISMA-P) 2015: elaboration and explanation. BMJ 2015;349:g7647.

24. Scale TN-O. adapted for cross-sectional studies. 
25. Berkey CS HD, Mosteller F, Colditz GA. A random-effects regression model for meta-analysis. Statistics in medicine. 1995;14(4):395-411.

26. LN DR. Meta-analysis in clinical trials. Control Clin Trials Control Clin Trials. 1986;7(177-88).

27. TJ FM. Transformations related to the angular and the square root. AnnMath Stats. 1950;21(4):60711.

28. Higgins JP TS. Quantifying heterogeneity in a meta-analysis. Statistics in medicine. Statistics in medicine 2002;21(11).

29. JL L. The role of the funnel plot in detecting publication and related biases in meta-analysis. Evidence-based dentistry. 2011;12(4):121-2.

30. Egger M DSG, Schneider M, Minder C. Bias in meta-analysis detected by a simple, graphical test. BMJ (Clinical research ed). 1997;315(7109):629-34.

31. Duval S T, R., . Trim and fill: a simple funnel-plot-based method of testing and adjusting for publication bias in meta-analysis. Biometrics 2000;56:455-63.

32. al He. The prevalence of depression and associated factors in Ethiopia: findings from the National Health Survey. International Journal of Mental Health Systems 2012;6(23):1-12.

33. al. Be. Depression and Its Help Seeking Behaviors: A Systematic Review and Meta-Analysis of Community Survey in Ethiopia. Depression Research and Treatment Volume 2018, Article ID 1592596, 11 pages https://doiorg/101155/2018/1592596.

34. Kefyalew Addis Alene ACAC, Emma S McBryde, Ernesto Jaramillo, Knut L $\neg$ onnroth, Debebe Shaweno , Amelia Gulliver , Kerri Viney Mental health disorders, social stressors, and health-related quality of life in patients with multidrug-resistant tubercu- losis: A systematic review and metaanalysis, Journal of Infection (2018), doi: 10.1016/j.jinf.2018.07.007.

35. Pachi A BD, Moussas G, Tselebis A. Psychiatric morbidity and other factors affecting treatment adherence in pulmonary tuberculosis patients. Tuberc Res Treat 2013; 2013:489865 [PubMed: 23691305].

36. Uplekar M WD, Lönnroth K, et al. WHO's new end TB strategy. Lancet. 2015; 385:1799-1801. [PubMed: 25814376].

\section{Tables}

Table 1. Characteristics of the studies 


\begin{tabular}{|l|l|l|r|r|r|r|r|}
\hline Author, Year & Study & Design & Tool & Response & Sample Cases & Prevalence \\
& & & & & & & \\
\hline Mulatu, 2019 & Setting & & & rate & size & & \\
\hline Molla, 2019 & Addis Ababa & Cross-Sectional & PHQ & 98.1 & 415 & 129 & 31 \\
\hline Dassa, 2019 & Oromia & Cross-Sectional & PHQ & 100 & 403 & 209 & 52 \\
\hline Tola, 2015 & Addis Ababa & Longitudinal & K-10 & 100 & 330 & 222 & 67 \\
\hline Adem, 2014 & Oromia & Cross-Sectional & K-10 & 89.5 & 222 & 44 & 20 \\
\hline Deribew, 2010 & Oromia & Cross-Sectional & K-10 & 80 & 124 & 68 & 55 \\
\hline Ambaw, 2017 & SNNP & Cross-Sectional & PHQ & 99.4 & 657 & 355 & 54 \\
\hline Duko, 2015 & SNNP & Cross-Sectional & HADS & 98.6 & 417 & 181 & 43 \\
\hline Ambaw, 2018 & SNNP & Cross-Sectional & PHQ & 98.6 & 648 & 349 & 54 \\
\hline Deribew, 2013 & Oromia & Longitudinal & K-10 & 78 & 97 & 18 & 19 \\
\hline Ambaw, 2019 & SNNP & Longitudinal & PHQ & 90.1 & 592 & 313 & 53 \\
\hline Tola, 2017 & Addis Ababa & Cross-Sectional & K-10 & 100 & 698 & 341 & 49 \\
\hline Ambaw, 2019 & SNNP & Longitudinal & PHQ & 90.1 & 299 & 146 & 49 \\
\hline Mohammed, 2018 & Addis Ababa & Cross-Sectional & DSM-IV & ----- & 346 & 95 & 27 \\
\hline Yadach, 2012 & Addis Ababa & Cross-Sectional & PHQ & 96.7 & 817 & 475 & 58 \\
\hline
\end{tabular}

Note: DSM-IV, K-10: Kessler Psychological/Mental Distress Scale, HADS: Hospital Anxiety and Depression Scale, PHQ-9: Patient Health Questionnaire-9

Table 2. Subgroup analyses by study: design, sample size, quality, publication year and setting. 


\begin{tabular}{|l|l|l|l|l|l|}
\hline Subgroup & $\begin{array}{l}\text { Number of } \\
\text { Studies }\end{array}$ & $\begin{array}{l}\text { Pooled } \\
\text { prevalence }\end{array}$ & $95 \% \mathrm{Cl}$ & $\mathrm{I}^{2}$ & P-value \\
\hline Study design & & & & & \\
\hline Cross-sectional & 11 & $46 \%$ & $38 \%-53 \%$ & 96.55 & $<0.001$ \\
\hline Longitudinal & 4 & $47 \%$ & $32 \%-62 \%$ & 96.36 & $<0.001$ \\
\hline Sample size & & & & & \\
\hline$<300$ & 7 & $42 \%$ & $27 \%-58 \%$ & 97.76 & $<0.001$ \\
\hline$\geq 300$ & 8 & $49 \%$ & $44 \%-55 \%$ & 93.12 & $<0.001$ \\
\hline Study quality & & & & & \\
\hline Fair & 4 & $31 \%$ & $20 \%-44 \%$ & 96.12 & $<0.001$ \\
\hline Good & 11 & $52 \%$ & $46 \%-58 \%$ & 94.49 & $<0.001$ \\
\hline Publication year & & & & & \\
\hline $2011-2015$ & 4 & $37 \%$ & $16 \%-61 \%$ & 98.08 & $<0.001$ \\
\hline $2016-2019$ & 11 & $49 \%$ & $43 \%-56 \%$ & 95.51 & $<0.001$ \\
\hline Study setting & & & & & \\
\hline Addis Ababa & 5 & $46 \%$ & $33 \%-60 \%$ & 97.98 & $<0.001$ \\
\hline Oromia & 5 & $42 \%$ & $23 \%-60 \%$ & 97.96 & $<0.001$ \\
\hline SNNP & 5 & $51 \%$ & $47 \%-55 \%$ & 73.97 & $<0.001$ \\
\hline
\end{tabular}

Note:- $95 \% \mathrm{Cl}$ represents the $95 \%$ Confidence Interval for prevalence and $\mathrm{I}^{2}$ represents the prevalence of true heterogeneity.

Table 3. Factors associated with depression among TB-patients in Ethiopia (pooled OR with 95\% Cl). 


\begin{tabular}{|l|l|l|}
\hline Factors & Number of studies & Pooled OR (95\%Cl) \\
\hline Age & 2 & $1.3(0.80-1.87)$ \\
\hline Female & 4 & $1.6(1.10-2.23)$ \\
\hline Low income & 3 & $3.0(1.16-4.92)$ \\
\hline HIV co-infection & 3 & $1.9(0.98-2.73)$ \\
\hline Substance use & 4 & $3.2(1.80-4.45)$ \\
\hline Poor social support & 4 & $1.9(0.77-3.13)$ \\
\hline Perceived stigma & 5 & $2.2(1.67-6.40)$ \\
\hline Extra pulmonary TB & 2 & $2.1(1.48-2.78)$ \\
\hline Intensive phase of treatment & 3 & $1.6(1.10-2.09)$ \\
\hline Note: Only those factors that had at least two studies were included in meta-analysis. \\
\hline
\end{tabular}

\section{Figures}




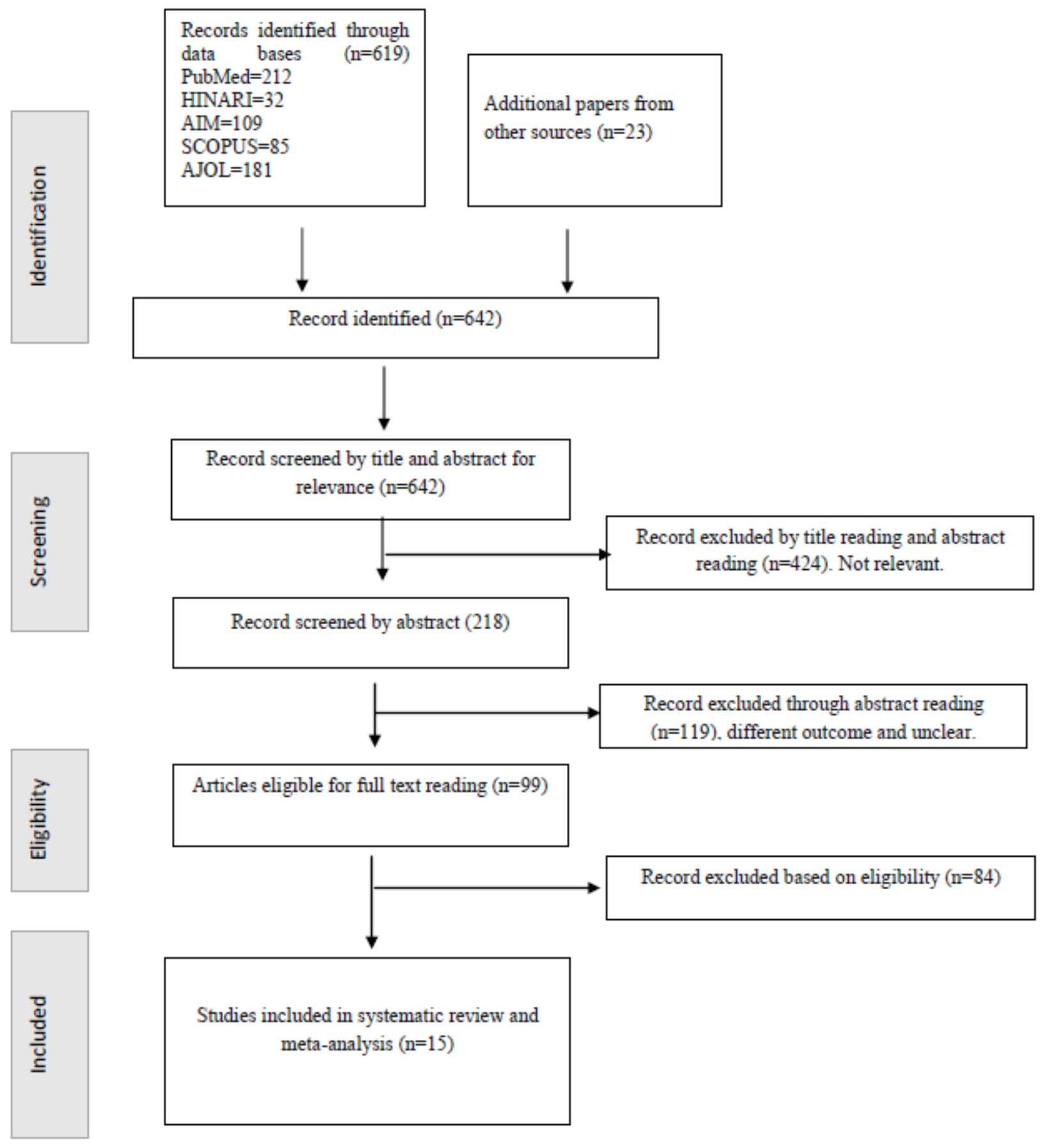

\section{Figure 1}

Flow diagram of included studies 


\begin{tabular}{|c|c|c|c|c|c|}
\hline Author & year & & & ES $(95 \% \mathrm{Cl})$ & $\begin{array}{l}\% \\
\text { Weight }\end{array}$ \\
\hline Mulatu & 2019 & \multirow{4}{*}{$\rightarrow-$} & $\rightarrow-$ & $0.63(0.58,0.68)$ & 6.72 \\
\hline Molla & 2019 & & & $0.31(0.27,0.36)$ & 6.75 \\
\hline Dassa & 2019 & & $\rightarrow-$ & $0.52(0.47,0.57)$ & 6.74 \\
\hline Tola & 2015 & & $\rightarrow-$ & $0.67(0.62,0.72)$ & 6.69 \\
\hline Adem & 2014 & \multirow[t]{3}{*}{$\rightarrow-$} & & $0.20(0.15,0.26)$ & 6.54 \\
\hline Deribew & 2010 & & $\longrightarrow$ & $0.55(0.46,0.63)$ & 6.21 \\
\hline Ambaw & 2017 & & $\rightarrow$ & $0.54(0.50,0.58)$ & 6.84 \\
\hline Duko & 2015 & \multirow[b]{3}{*}{$\hookleftarrow$} & & $0.43(0.39,0.48)$ & 6.75 \\
\hline Ambaw & 2018 & & $\rightarrow$ & $0.54(0.50,0.58)$ & 6.84 \\
\hline Deribew & 2013 & & & $0.19(0.12,0.27)$ & 6.02 \\
\hline Ambaw & 2019 & \multirow{5}{*}{$\rightarrow-$} & $\rightarrow-$ & $0.53(0.49,0.57)$ & 6.83 \\
\hline Tola & 2017 & & & $0.49(0.45,0.53)$ & 6.85 \\
\hline Ambaw & 2019 & & & $0.49(0.43,0.54)$ & 6.65 \\
\hline Mohammed & 2018 & & & $0.27(0.23,0.32)$ & 6.70 \\
\hline Yadach & 2012 & & $\rightarrow$ & $0.58(0.55,0.61)$ & 6.88 \\
\hline \multicolumn{2}{|c|}{ Overall $\left(l^{\wedge} 2=96.33\right)$} & $=0.00)$ & & $0.46(0.40,0.53)$ & 100.00 \\
\hline & & $\begin{array}{l}1 \\
.25\end{array}$ & $\begin{array}{c}1 \\
.75\end{array}$ & 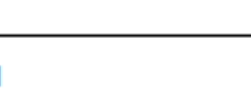 & \\
\hline
\end{tabular}

Figure 2

Forest plot presenting the prevalence of depression using random effect models with $95 \% \mathrm{Cl}$. 


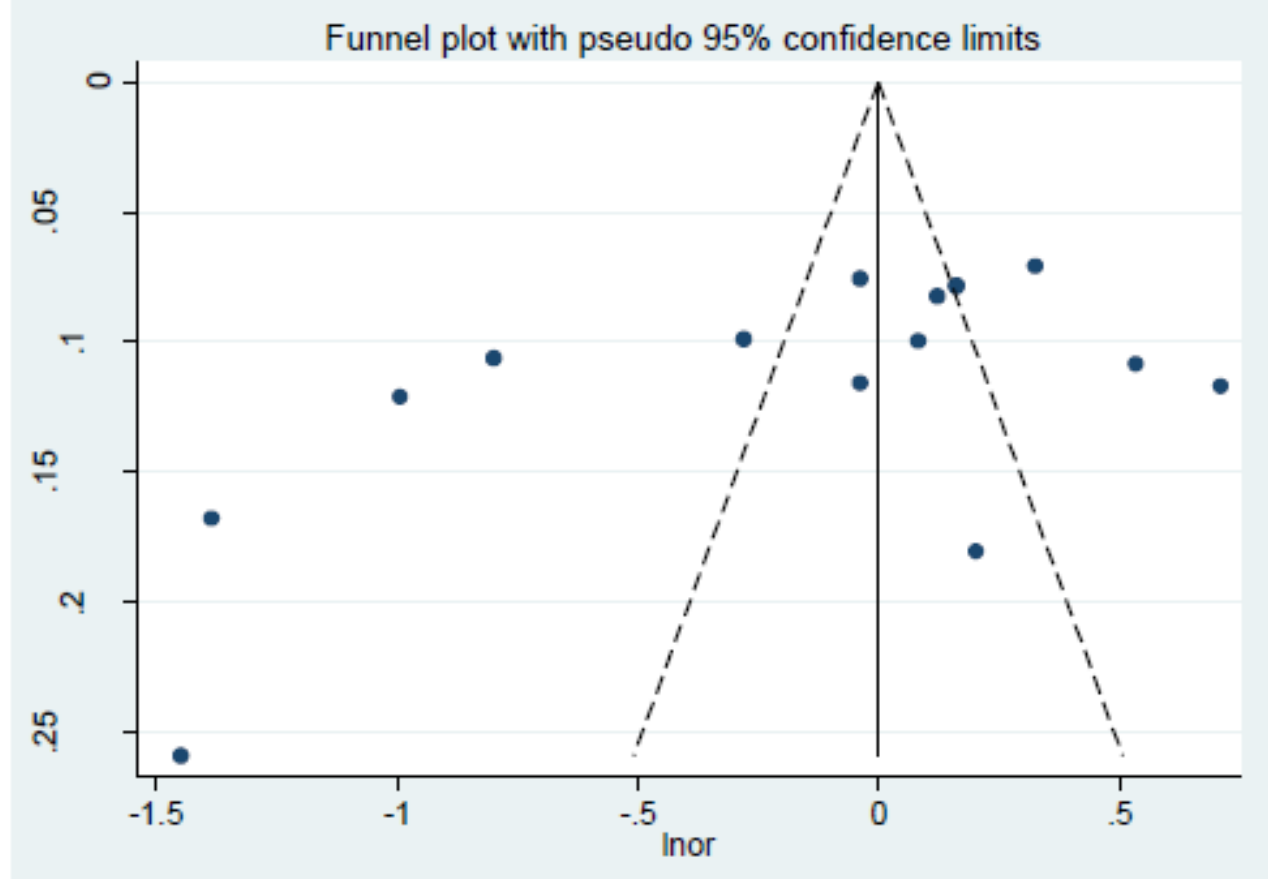

Figure 3

Funnel plot with pseudo $95 \%$ confidence interval that investigated the publication bias of the pooled prevalence of depressive symptoms.

\section{Supplementary Files}

This is a list of supplementary files associated with this preprint. Click to download.

- SupplementaryfilesPRISIMACHECKLIST.pdf 